\title{
Hacia un modelo integrador de la tutoría en los diferentes niveles educativos
}

\section{Towards an integrative tutoring model across different educational levels}

\author{
Manuel Álvarez González ${ }^{1}$ \\ alvarez.m@ub.edu \\ Universidad de Barcelona, España
}

\begin{abstract}
Resumen:
El principal objetivo de este trabajo es presentar un modelo integrador de tutoría para los diferentes niveles educativos. Este se ha estructurado en dos grandes apartados: un primer apartado donde se desarrollan una serie de aspectos previos, que permitirán contextualizar la tutoría en el momento presente (la acción tutorial como un factor de calidad de la educación, las fortalezas y debilidades del modelo tutorial actual, la función docente y la función tutorial, los nuevos roles y funciones de la acción tutorial y el marco conceptual de la tutoría); y en un segundo apartado se plantea el modelo integrador de la tutoría, destacando las razones que lo justifican, las características que lo definen, los objetivos y dimensiones a desarrollar, las estrategias de intervención y el modelo organizativo más adecuado para su puesta en práctica.
\end{abstract}

Palabras clave:

Educación; calidad educativa; tutoría; orientación; función docente; función tutorial; modelo integrador de tutoría.

\begin{abstract}
:
The main objective of this study is to present an integrative tutoring model for the different educational levels. It is has been structured in two main sections: a first section where a series of preliminary aspects are discussed, which will allow contextualizing tutoring as conceived nowadays (tutorial action as a quality factor of education, strengths and weaknesses of current tutorial models, the teaching and the tutorial functions, the new roles and functions of tutorial action and the conceptual framework underlying tutoring). The second section will present an integrative tutoring model highlighting the reasons that justify it, the characteristics that define it, its objectives and dimensions and the intervention strategies and the organizational model most suitable for its implementation.
\end{abstract}

\section{Key words:}

Education; educational quality; educational levels; tutoring; guidance; teaching function; tutorial function; integrative tutoring model.

1 Dirección para correspondencia (correspondence address):

Manuel Álvarez González. Dpto. de Métodos de Investigación y Diagnóstico en Educación. Facultad de Pedagogía. Universidad de Barcelona. Avda. Línea Eléctrica, 9, 2. ${ }^{\circ}-4{ }^{\text {a }} 08940$ Cornellá de Llobregat, Barcelona (España). 


\begin{abstract}
Résumé:
Le principal objectif de ce travail est de présenter un modèle intégrateur d'action de tutorat pour les différents niveaux éducatifs. Ce dernier a été organisé sur deux grands axes principaux. Un premier axe où se développe une série d'aspects préliminaires qui permettront de contextualiser l'action de tutorat dans l'instant présent (l'action de tutorat comme facteur de qualité éducative, les points forts et les points faibles de ce modèle, la fonction enseignante et la fonction de tutelle, les nouveaux rôles et nouvelles fonctions de l'action de tutorat, ainsi que le cadre conceptuel de l'action de tutorat). Dans le deuxième axe, il y aura une mise en question de ce modèle intégrateur. Les raisons qui justifient son usage sont mises en relief, ainsi que les caractéristiques qui le définissent, les objectifs et les dimensions qui devront être développés, les stratégies d'intervention et le modèle organisationnel le plus adéquat pour sa mise en pratique.
\end{abstract}

\title{
Mots clés:
}

Éducation; qualité éducative; action de tutorat; orientation; fonction enseignante; fonction de tutelle; modèle intégrateur de l'action de tutorat.

Fecha de recepción: 28-2-2017

Fecha de aceptación: 18-4-2017

\section{Introducción}

La acción tutorial es un componente básico y fundamental del proceso educativo y un elemento nuclear de la práctica docente en los diferentes niveles educativos. La tutoría se ha de entender como un proceso continuo y sistemático que facilite los procesos de aprendizaje y la toma de decisiones, que atienda la diversidad y la inclusión, que potencie el desarrollo personal y que asesore sobre las opciones educativas y laborales. La labor de la orientación y la tutoría ha de servir para integrar los diferentes aspectos que conforman el proceso madurativo y formativo del alumnado, prestando especial atención a su desarrollo integral. La tutoría es un espacio para el desarrollo integral del alumnado en todos sus aspectos y, al mismo tiempo, se ha convertido en uno de los factores de calidad de la educación. De ahí la necesidad de plantear la acción tutorial como una acción cooperativa, asumida por los diferentes agentes educativos y que requiere de la tarea coordinada de las personas e instituciones que intervienen en la educación. Para ello hemos de ir hacia un nuevo enfoque más comprensivo e integral de la tutoría. En este trabajo expondremos en qué consiste este modelo integrador de la tutoría en los centros educativos. 


\section{Contextualización de la tutoría}

Hay una serie de aspectos que se han de tener en cuenta si queremos contextualizar y luego implantar un modelo integrador de tutoría.

\subsection{La acción tutorial como un factor de calidad de la educación}

Los diferentes autores y organismos consultados (Thayer, 1994; Burgess, 1994; Rodríguez Espinar, 1995, 2002, 2006, 2015; Delors, 1996; De Miguel, 1997; Comisión Europea, 2000; Sanz, 2001; Sarramona, 2002; Álvarez Pérez, 2002; Braslavsky, 2004; Gerogiannis y Fitsilis, 2010; Arias Aparicio y Gentile, 2011, UNESCO, 2004, 2014, 2015) coinciden en señalar que la calidad tendrá que ver con la adecuación de la institución para alcanzar los objetivos que le son propios. No puede entenderse la calidad de la educación, si no se tiene en cuenta la propia institución y su entorno próximo. Una institución educativa de calidad ha de ofertar lo que sus usuarios necesitan: hacer bien aquello que se tiene que hacer (Rodríguez Espinar, 1997, 2006).

La calidad de la educación se ha convertido en una cuestión elemental y prioritaria en nuestro entorno (Unión Europea). Las conclusiones de las diferentes cumbres europeas (Lisboa, 2000; Barcelona, 2002; y últimamente Bruselas, 2015; Bratislava, 2016) destacan el papel fundamental que desempeña la educación y la formación en un mundo cada vez más exigente y competitivo. Nuestro sistema educativo ha de ser cada vez más eficaz y eficiente si quiere dar respuesta a las problemáticas que más nos preocupan como el fracaso académico, la desmotivación, el absentismo, las conductas antisociales, el desempleo, etc. Calidad de la educación no puede identificarse únicamente con una mejor preparación intelectual del alumnado (aprender hacer y aprender a saber) sino que se ha de ocupar también de aquellas competencias que hacen referencia a la dimensión personal, socioemocional y profesional (aprender a ser y aprender a convivir) e intentar desarrollarlas en los programas educativos (Álvarez González, 2004; Rodríguez Espinar, 2006).

Un sistema educativo tiende a la calidad o excelencia en la medida que sea capaz de: a) estimular la participación intra e intercentros y con el entorno social (comunicación); b) fomentar la formación y la motivación de los diferentes agentes educativos; c) intentar afrontar el fracaso escolar de una forma comprensiva y dotar al alumnado de competen- 
cias para aprender a aprender; d) familiarizarse en el uso de las nuevas tecnologías que faciliten la interacción y el intercambio de ideas y materiales entre profesorado, alumnado y alumnado entre sí (aprendizaje cooperativo); e) promover la capacidad para innovar y estar en constante adaptación con la nueva realidad (agente de cambio); f) adoptar un compromiso firme con la evaluación para mejorar aquellos aspectos que son susceptibles de mejora; g) favorecer un mayor conocimiento de sí mismo y del entorno que facilite el proceso de toma de decisiones a lo largo de la vida; h) facilitar la ayuda y asesoramiento a través de una intervención orientadora y tutorial a todos los agentes educativos (Álvarez González, 2008).

En las últimas décadas el Ministerio de Educación y Ciencia (MEC) ha Ilevado a cabo distintas iniciativas orientadas a la mejora de la calidad de la educación a través de las diferentes Leyes Educativas (Tabla 1).

\section{Tabla 1. Iniciativas de mejora de la calidad de la educación.}

\begin{tabular}{ll}
\hline Leyes educativas & \multicolumn{1}{c}{ Iniciativas de mejora de la calidad } \\
\hline LOGSE (1990) y & "77 medidas para la calidad educativa", agrupadas en torno \\
LOPE (1995) & a seis ámbitos diferenciados (valores, igualdad, centros, di- \\
& rección, profesorado e inspección). \\
& Planes anuales de mejora para los centros educativos: De- \\
& finición e implantación de planes anuales de mejora en \\
& Colegios e Institutos; adaptación y publicación del modelo \\
& europeo de Gestión de calidad en la Administración edu- \\
& cativa y periférica; y formación dirigida a candidatos a di- \\
& rectivos escolares; directores en ejercicio e inspectores de \\
& educación. \\
\hline LOCE (2002) & Propone una serie de medidas para la mejora de la calidad \\
& de la educación en torno a cinco ejes: la cultura del esfuer- \\
& zo, los procesos de evaluación, sistema de oportunidades \\
& de calidad para todos, profesorado, autonomía de los cen- \\
& tros. Esta ley no llegó a implantarse. \\
\hline LOE (2006) & lantea medidas para paliar la situación de fracaso esco- \\
& Orientación y Apoyo (PROA), Programas de Aprendizaje \\
& Básico (PAB) y Programas de Cualificación Profesional Ini- \\
Cial (PCPI), entre otros.
\end{tabular}

LOMCE (2013...) Uno de sus principios es "proporcionar una educación de calidad, donde el objetivo es mejorar los resultados generales y reducir el abandono temprano". 


\begin{tabular}{ll}
\hline Leyes educativas & \multicolumn{1}{c}{ Iniciativas de mejora de la calidad } \\
\hline LOU (2001, & En la exposición de motivos (apartado V) se expone que \\
"una) & por de las principales innovaciones de la Ley viene dada \\
& nismos externos de evaluación de su calidad, conforme a \\
& criterios objetivos y procedimientos transparentes. Para ello \\
& se crea la Agencia Nacional de Evaluación de la Calidad \\
& y Acreditación. Esta promoverá y garantizará la calidad de \\
& las universidades, objetivo esencial de la política universi- \\
& taria". \\
\hline
\end{tabular}

El mandato legal a favor de una educación de calidad es evidente, otra cosa es si se han puesto los medios para desarrollar esa educación de calidad y de equidad, claramente no ha sido así. Por un lado las iniciativas propuestas por la Administración educativa no contemplan, de forma explícita, la orientación y la acción tutorial como un factor de calidad, pero sí de forma implícita con algunas medidas e iniciativas como la implantación en los centros de los Servicios de orientación en primaria, los Departamentos de orientación en secundaria, la Coordinación de tutoría y los Servicios de orientación universitaria en la educación superior. Y, por otro lado, la actual situación de crisis económica no ha facilitado el que se pongan los medios necesarios; es más, se han acentuado las desigualdades educativas y ha aumentado la pobreza infantil. Estudios realizados por González Bueno, Bello y Arias (2012), Albaigés y Martínez (2013), UNICEF (2013), destacan que los niños son los más vulnerables ante esta crisis porque se ha reducido significativamente el gasto público (falta de becas, ayudas para libros, ayudas de comedor) y se han visto menguados los ingresos familiares. A nivel universitario han subido las tasas de matrícula y han disminuido las becas. A pesar del momento que vivimos, no debemos olvidar que la orientación y la tutoría son unas medidas de prevención importantísimas para mejorar la calidad de la educación. Para asumir de forma plena la educación se necesita la ayuda y el aporte de la orientación y la acción tutorial.

\subsection{Fortalezas y debilidades del modelo tutorial actual}

Durante estas últimas décadas se han dado pasos en el proceso de implantación de la orientación y la tutoría en los diferentes niveles educativos, pero no han sido suficientes porque no han aportado los resultados 
esperados. Para analizar las fortalezas y debilidades del modelo tutorial actual nos servimos de las dimensiones de análisis que nos propone Montserrat Pera (2011) en un excelente trabajo sobre tutoría (Tabla 2).

Tabla 2. Fortalezas y debilidades del modelo tutorial actual.

\begin{tabular}{|c|c|c|}
\hline Dimensión & Fortalezas & Debilidades \\
\hline $\begin{array}{l}\text { Marco ins- } \\
\text { titucional }\end{array}$ & $\begin{array}{l}\text { Política institucional favorecedora } \\
\text { de un proceso de cambio y mejora } \\
\text { de la docencia. } \\
\text { Reconocimiento del tutor. } \\
\text { PAT general del centro. }\end{array}$ & $\begin{array}{l}\text { Falta de planificación y diseño } \\
\text { de PATs en algunos centros. } \\
\text { Falta de tradición en procesos } \\
\text { de orientación y seguimiento } \\
\text { del estudiante. }\end{array}$ \\
\hline $\begin{array}{l}\text { Planifica- } \\
\text { ción del } \\
\text { PAT }\end{array}$ & $\begin{array}{l}\text { Concreción del PAT de centro a la } \\
\text { realidad concreta donde se aplica. } \\
\text { Soporte para desarrollar el PAT. } \\
\text { Estrategia de organización e inte- } \\
\text { gración de la tutoría. }\end{array}$ & $\begin{array}{l}\text { Falta de organización para } \\
\text { que la tutoría sea operativa. } \\
\text { Deficiente coordinación de la } \\
\text { tutoría. } \\
\text { Escasa relevancia de la tuto- } \\
\text { ría de asignatura. } \\
\text { Falta de tiempo y espacio } \\
\text { para la tutoría. }\end{array}$ \\
\hline $\begin{array}{l}\text { Acompa- } \\
\text { ñamiento } \\
\text { de las } \\
\text { tutorías }\end{array}$ & $\begin{array}{l}\text { Buena predisposición inicial. } \\
\text { Cierto impacto cuando se lleva a } \\
\text { cabo. } \\
\text { Una mínima coordinación docente. } \\
\text { Eficacia y coherencia de la acción } \\
\text { tutorial. }\end{array}$ & $\begin{array}{l}\text { Escasa respuesta y asistencia } \\
\text { de los tutores/as. } \\
\text { Poca implicación del profeso- } \\
\text { rado en la acción tutorial. } \\
\text { Insuficiente coordinación de } \\
\text { los tutores. }\end{array}$ \\
\hline $\begin{array}{l}\text { Formación } \\
\text { de los } \\
\text { tutores }\end{array}$ & $\begin{array}{l}\text { Asistencia de los tutores a cursos } \\
\text { de formación. } \\
\text { Capacitación del profesorado. } \\
\text { Plan de formación orientado a la } \\
\text { demanda tutorial. }\end{array}$ & $\begin{array}{l}\text { Falta de una formación inicial } \\
\text { y contínua adaptada al perfil } \\
\text { de los tutores. } \\
\text { Desconocimiento y escasa } \\
\text { formación de los procesos de } \\
\text { orientación y tutoría por par- } \\
\text { te del profesorado. }\end{array}$ \\
\hline $\begin{array}{l}\text { Difusión a } \\
\text { los } \\
\text { estudian- } \\
\text { tes }\end{array}$ & $\begin{array}{l}\text { Acciones de difusión presenciales } \\
\text { y virtuales con estudiantes. } \\
\text { Información vía web, correo elec- } \\
\text { trónico. }\end{array}$ & $\begin{array}{l}\text { Desconocimiento del PAT por } \\
\text { parte de algunos estudiantes. } \\
\text { Expectativas de los estudian- } \\
\text { tes que no se cumplen en el } \\
\text { PAT. }\end{array}$ \\
\hline $\begin{array}{l}\text { Desarro- } \\
\text { llo de la } \\
\text { acción } \\
\text { tutorial }\end{array}$ & $\begin{array}{l}\text { Formación integral del estudiante. } \\
\text { Actitud e interés del tutor. } \\
\text { Comunicación con los estudiantes. } \\
\text { Mayor conocimiento de sus nece- } \\
\text { sidades, intereses y problemáticas. }\end{array}$ & $\begin{array}{l}\text { Frecuencia en la comunica- } \\
\text { ción y la participación. } \\
\text { Poca asunción del rol del tu- } \\
\text { tor por parte del profesorado. } \\
\text { Poca flexibilidad en el hora- } \\
\text { rio de tutorías. }\end{array}$ \\
\hline
\end{tabular}




\begin{tabular}{|c|c|c|}
\hline Dimensión & Fortalezas & Debilidades \\
\hline $\begin{array}{l}\text { Herra- } \\
\text { mienta vir- } \\
\text { tual para } \\
\text { la tutoría }\end{array}$ & $\begin{array}{l}\text { Facilidad y funcionalidad del uso } \\
\text { de las TIC en la tutoría. } \\
\text { Mayor flexibilidad, más intercam- } \\
\text { bio de información, comunica- } \\
\text { ción y recursos. } \\
\text { Seguimiento más personalizado } \\
\text { del estudiante. }\end{array}$ & $\begin{array}{l}\text { Poco uso de las TIC por parte } \\
\text { de los tutores. } \\
\text { Falta de tiempo por parte del } \\
\text { profesorado. } \\
\text { Escaso porcentaje de estu- } \\
\text { diantes que utilizan el espa- } \\
\text { cio virtual. }\end{array}$ \\
\hline
\end{tabular}

A modo de síntesis, podríamos destacar una serie de aspectos que definen el modelo actual de la orientación y la tutoría en los diferentes niveles educativos (Álvarez González y Álvarez Justel (2015): a) la tutoría no ha estado integrada, de forma plena, en la formación del estudiante; b) ha carecido en ocasiones de objetivos y contenidos concretos; c) no existe una gran disponibilidad de horarios por parte del profesorado y alumnado para la tutoría; d) en algunos casos la tutoría se convierte en algo burocrático (servicio social, becas, etc.); e) a veces se confunde el acompañamiento académico tutorial con un espacio para el desahogo emocional; f) Poco uso de las TIC por parte de los tutores; g) en muchas ocasiones se produce una falta de capacitación continua y específica de los tutores; h) y frecuentemente se manifiesta una falta de interés del alumnado y una falta de tiempo del profesorado por la tutoría.

Por lo tanto, hemos de reorientar el modelo tutorial a través de una profunda redefinición de la función docente y de la función tutorial del profesorado.

\subsection{La función docente y la función tutorial}

La práctica docente ha de asumir la dimensión orientadora y tutorial. La función tutorial y orientadora son los componentes básicos de una actividad docente que ha de asegurarnos una educación integral y personalizada. Ha de quedar claro que la tutoría es un elemento inherente a la función docente y al currículum. Todo profesor está implicado en la acción tutorial, independientemente de que se le asigne un grupo de alumnos como tutor. Para ello, es necesario superar la tradición del docente como impartidor de conocimientos y asumir las funciones esencialmente formativas. Ahora bien no se trata de convertir a los docentes en orientadores, sino de volver más explícita la función que cada profesorado desarrolla, de forma espontánea, como facilitador del proceso de 
desarrollo integral del alumnado. Para esta labor orientadora y tutorial el tutor deberá contar con la ayuda de la comunidad educativa, especialmente con los especialistas de orientación (servicios internos y externos al centro) y con la colaboración del alumnado de los últimos cursos (peer tutoring), sobre todo en la educación superior (Álvarez González, 2006).

Así pues, podemos estructurar la acción tutorial y orientadora en tres niveles de intervención: a) el primer nivel corresponde a la acción tutorial y orientadora que ha de hacer el profesorado (tutoría de asignatura) y el tutor (tutoría de acompañamiento); b) un segundo nivel referido al asesoramiento, formación y soporte técnico a la tutoría (Departamento y/o Servicios de orientación en el centro); c) y un tercer nivel centrado más en la colaboración en aspectos específicos de la acción orientadora (Servicios de orientación externos de la comunidad).

\subsection{Los nuevos roles y funciones de la orientación y acción tutorial}

La realidad socio-educativa ha de afrontar la interculturalidad, los avances tecnológicos, el aumento del fracaso escolar, el absentismo, el abandono, la inadaptación social, la globalización, etc., donde la presencia de la orientación y la tutoría son absolutamente necesarias. Esta realidad al estar en constante cambio requiere también un cambio en los roles y funciones de la orientación y la tutoría.

La práctica orientadora y tutorial se podría concretar en una serie de roles y funciones (Ginter, Salise y Press, 1990; Welch y McCarrol, 1993; Sanz y Sobrado, 1998; Watts y Esbroeck, 1998; Álvarez González y Rodríguez Espinar, 2000; Marín y Rodríguez Espinar, 2001; ASCA, 2005; De la Cruz, García y Abreu, 2006; Álvarez González y Bisquerra, 2012; Álvarez González y Álvarez Justel, 2013; González Palacios y Avelino Rubio, 2016):

- Asesoramiento individual y en pequeño grupo (counseling). Se ha de asumir el asesoramiento individual y grupal con los diferentes agentes de intervención (alumnado, familia, profesorado); se han de afrontar los diferentes contenidos de tipo personal, familiar, escolar, profesional, laboral, social, problemáticas interpersonales, etc.; y se ha de dar un mayor protagonismo a las funciones de carácter preventivo, de desarrollo y de intervención social.

- Coaching. Es una dimensión de la orientación y la tutoría que pro- 
porciona ayuda para el conocimiento personal y profesional del alumnado. Los orientadores y tutores deben familiarizarse con el coaching para el liderazgo, para el cambio, para la relación personal, para el desempeño profesional, etc. En el coaching se da información y asesoramiento al alumnado para que éste pueda mejorar sus competencias.

- Consulta a otros agentes implicados en la intervención orientadora y tutorial. La consulta comporta un trabajo en equipo (colaboración, coordinación, intercambio) con un mayor protagonismo del rol de consultor colaborativo en detrimento del rol de consultor experto. Esta consulta colaborativa supone un cambio de actitud en los profesionales de la orientación, de la educación y de la formación en general.

- Coordinación entre todos los agentes implicados en la intervención y entre todos los servicios, organismos e instituciones que actúan en una población. Se impone un modelo de actuación centrado en un plan de intervención integral y coordinado desde el nivel local (Municipio). Es absolutamente imprescindible establecer una eficaz coordinación entre todos los servicios, organismos, etc., que afrontan la acción orientadora y tutorial en un contexto determinado.

- Currículum. Integración paulatina de la orientación y la tutoría en el currículum, utilizando diferentes estrategias (asignaturas, tutoría, integración disciplinar e interdisciplinar) hasta alcanzar la infusión plena y considerar la orientación y la tutoría como una actividad más en la tarea escolar. Los profesionales de la orientación son educadores y los profesionales de la educación son orientadores que comparten con el resto de agentes de la institución (alumnado, familia, etc.) la responsabilidad del desarrollo integral del alumnado.

- Tecnologías de la información y la comunicación. Estamos en la sociedad de la información y de la comunicación y los profesionales de la orientación y de la educación no deben obviar las nuevas tecnologías (Programas informáticos, páginas y portales web, videoconferencia, foro, blog, WQ, wikis, moodle, Herramientas 2.0, etc.) como una herramienta útil en la intervención orientadora y tutorial. Estas nos pueden proporcionar estrategias y sistemas más dinámicos, participativos e interactivos en los procesos de apren- 
dizaje, la información (personal, académica y ocupacional), los procesos de toma de decisiones, los itinerarios de inserción sociolaboral, etc. Como señala Pantoja (2006, 2010), todos estos recursos tecnológicos hacen más continuado e interactivo el proceso tutorial.

- Agente de cambio. El tutor ha de concebir el contexto como el medio adecuado para planificar y desarrollar su acción orientadora y tutorial de forma colaborativa y desde una perspectiva reflexiva que permita ir reconstruyendo y cambiando dicho contexto.

\subsection{Marco conceptual de la tutoría}

Podríamos definir la acción tutorial como la acción formativa de orientación, ayuda y mediación que el profesor-tutor y el resto del equipo docente realiza con su alumnado a nivel individual y grupal en el ámbito personal, escolar, profesional y social, al mismo tiempo que ejercen su función docente. Esta acción formativa ha de dirigirse también y ha de contemplar la colaboración del resto de agentes educativos, particularmente de las familias (Álvarez González, 2004; Sanz, 2010; Álvarez González y Bisquerra, 2012). La acción tutorial es una tarea de toda la comunidad educativa, donde el tutor tiene un papel preponderante como responsable de coordinar, dinamizar y llevar a cabo la función tutorial de su grupo con la ayuda y colaboración del resto del profesorado, orientador, familia e institución.

Para poder desarrollar las diferentes áreas o contenido de la tutoría es necesario disponer de unos modelos teóricos que van a servir de guía para la acción y para fundamentar la propia intervención. El tutor puede fundamentar su intervención en uno o varios enfoques que van desde planteamientos racionales hasta planteamientos cognitivos y de desarrollo, pasando por planteamientos no directivos. Concretamente en el ámbito de la educación no universitaria se puede hablar de modelos: racionales, no directivos, cognitivos y de desarrollo y eclécticos (Álvarez González, 2004; Álvarez González y Bisquerra, 2012), y en el ámbito de la educación superior de modelos: académicos, de desarrollo personal, de desarrollo profesional y sistémicos (Rodríguez Espinar y otros, 2004). Dichos modelos lo que nos van a permitir es confeccionar nuestra teoría o enfoque de la intervención tutorial. 


\section{Hacia un modelo integrador de la tutoría}

Por todo lo comentado anteriormente, es importante dar el paso hacia un modelo integral de la acción tutorial y la orientación que incluya los distintos aspectos que conforman el desarrollo integral del alumnado (personal, académico, profesional, social, emocional), en sus diferentes modalidades de tutoría (tutoría académica o de asignatura, la tutoría de la carrera o de acompañamiento y la tutoría de asesoramiento personal, además de la tutoría de iguales y la tutoría de prácticum), con la implicación de toda la comunidad educativa (alumnado, profesorado, familia, orientadores) y en todos sus niveles educativos (infantil, primaria, secundaria y educación superior).

La acción tutorial ha de ayudar, orientar, acompañar al alumnado desde su más temprana edad, facilitándole la madurez y la formación integral necesarias para desenvolverse con éxito en cualquier situación de la vida. Si hemos hablado de la conveniencia de una educación integral que prepare para la vida, también hemos de plantear una tutoría integral que vaya en consonancia con esa nueva concepción de la educación. Hay una serie de razones que justifican la necesidad de esta tutoría integradora (Álvarez González y Álvarez Justel (2012, 2014, 2015):

1) El modelo tutorial existente no ha dado los resultados que se esperaban porque desde sus inicios se ha visto como algo desligado de la función docente y del desarrollo académico y curricular del alumnado.

2) La estrategia utilizada en su elaboración y desarrollo no ha sido la adecuada porque se han adoptado estrategias y metodologías poco implicadoras y escasamente motivadoras.

3) Se ha centrado fundamentalmente en algunos aspectos de la dimensión académica (aclarar dudas y revisar trabajos, entre otros aspectos).

4) El profesor sigue siendo un actor esencial en los procesos de aprendizaje del alumnado. El docente tiene un papel primordial para individualizar, supervisar y evaluar el trabajo autónomo del alumnado.

5) Se ha de crear un espacio de convergencia docente y tutorial si se quiere desarrollar el proceso tutorial de forma operativa y eficiente.

6) Se ha de acompañar al estudiante en su proceso formativo para 
que adquiera una formación integral en todos sus aspectos (personal, académica, social, profesional y emocional).

7) La tutoría es multidisciplinar e interdisciplinar que implica a todos los agentes educativos y de forma colaborativa.

8) Ha carecido de objetivos y contenidos concretos. En la mayoría de las ocasiones se ha guiado por el voluntarismo del tutor y con una deficiente concreción.

9) Poco uso de las TIC por parte de los tutores y escaso número de estudiantes que utilizan el espacio virtual.

10) Es necesario un cambio de actitud por parte de la institución educativa y de los diferentes agentes educativos (profesorado, alumnado, familias, etc.). En definitiva, se ha de redefinir la función docente y tutorial del profesorado con el apoyo de la institución.

Una vez comprobada la necesidad de un modelo integrador de la tutoría ¿qué características lo definen? (Álvarez González, 2013, 2014; Álvarez González y Álvarez Justel, 2014) (tabla 3).

\section{Tabla 3. Características que definen el modelo integrador de tutoría.}

Características que definen este modelo integrador de tutoría

- Ha de estimular el desarrollo integral del alumnado a nivel personal, académico, social y profesional. El mismo alumnado reclama una participación en el espacio tutorial que favorezca su desarrollo integral.

- Se ha de convertir en un modelo de acompañamiento integral al alumnado a nivel institucional y reconocido por todos.

- La tutoría ha de considerarse como algo inherente a la educación y ha de estar integrada en el currículum, si queremos que se convierta en uno de los factores de calidad de la educación y así producir un efecto sinergia.

- Ha de facilitar la adquisición y el desarrollo de competencias personales y profesionales para la construcción del proyecto de vida.

- Ha de concebir la orientación y la tutoría como una acción multidisciplinar e interdisciplinar para afrontar los procesos de acompañamiento del alumnado que le ayude a planificar su proyecto de vida y fomentar el éxito.

- Ha de permitir una mejora en el proceso de acompañamiento del alumnado.

- Tanto el profesorado como el alumnado necesitan tiempos y espacios compartidos para desarrollar su acción tutorial.

- La institución educativa tiene la responsabilidad de identificar los cambios necesarios para una educación integral del alumnado (espacios de convergencia docente y tutorial).

- Se ha de crear una política institucional que regule y gestione la acción tutorial como una tarea integrada en el modelo educativo de la institución. . 
Un plan institucional de tutoría en los diferentes niveles educativos (desde educación infantil a la educación superior), que debe desarrollarse a lo largo de los distintos cursos y niveles (programación horizontal y vertical).

- Ha de ser un modelo adaptado a las necesidades del alumnado y a la propia institución.

- Se ha de potenciar el uso de las TIC en la tutoría para fomentar el intercambio, la participación, la interacción, los recursos y un seguimiento más personalizado del alumnado.

- Y requiere de un plan de formación y capacitación de todo el profesorado y del resto de agentes implicados. Para ello, primero se les ha de informar de su nueva labor, después se les ha de implicar, motivar y finalmente se les ha de formar.

\subsection{Objetivos de este modelo tutorial}

Los objetivos han de ir en consonancia con las características que definen este modelo integrador de la tutoría (Tabla 4).

Tabla 4. Objetivos del modelo integral de tutoría.

\section{Objetivos del modelo integral de tutoría}

- Fomentar el desarrollo integral del alumnado a lo largo de su proceso formativo.

- Favorecer la integración y la adaptación del alumnado al nuevo curso académico, etapa, ciclo.

- Acompañar al alumnado en su proceso de aprendizaje (enfoques, estilos, estrategias, metodología, dificultades de aprendizaje, abandono, fracaso escolar, etc.).

- Promover el desarrollo personal y social (conocimiento de sí mismo y de los demás, autonomía, autoestima, conciencia y regulación emocional, competencia social, habilidades de vida, habilidades sociales, etc.).

- Estimular el desarrollo profesional (toma de decisiones, proyecto profesional).

- Proporcionar atención a la diversidad y a una adecuada inclusión en el aula y en el centro (adaptaciones curriculares y metodológicas, agrupamientos flexibles, planificación multinivel, inclusión social y académica).

- Facilitar los procesos de transiciones académicas y laborales (familia-escuela, infantil-primaria, primaria-secundaria, secundaria-educación superior, educación superior-mundo laboral).

- Contribuir a la adecuada relación e interacción entre los distintos integrantes de la comunidad educativa (profesorado, alumnado, familia y entorno social).

- Posibilitar la conexión con otros servicios de apoyo al alumnado y a la tutoría (servicios de orientación, servicios sociales, servicios sanitarios, servicios de empleo, etc.).

- Potenciar el uso de las TIC. 


\subsection{Dimensiones de la tutoría integral}

En la siguiente tabla (Tabla 5) se presentan las áreas o dimensiones de una intervención integradora de la acción tutorial, teniendo en cuenta los diferentes niveles educativos. Para ello nos servimos de los trabajos realizados por Tracey y Shea, 2000; Rodríguez Espinar y otros, 2004; Arbizu, Lobato y del Castillo, 2005; De la Cruz, García y Abreu, 2006; Stevenson, 2006; McQuillan \& Coleman, 2008; Álvarez Pérez y González, 2009; García Pérez, 2010; Álvarez González, 2006, 2009, 2013; Barna\&Brott, 2011; Hill, 2012; Hillsdale, 2012; Álvarez González y Álvarez Justel, 2014, 2016; Hamlin y Kidder, 2015; McLelland\& Galton, 2015; González Palacios y Avelino Rubio, 2016; y Organismos como el Gobierno de Navarra, 2001; Public Schools of North Carolina, 2001; Texas Public School, 2004; The Education Bureau HKSAR, 2006; Comunidad de Madrid, 2008; ASCA, 2005; NACADA, 2008; Connecticut State Departament of Education (2014), entre otros.

Tabla 5. Dimensiones de la tutoría integral por niveles educativos.

\begin{tabular}{|c|c|c|}
\hline Infantil y primaria & Secundaria & Educación superior \\
\hline $\begin{array}{l}\text { Desarrollo del proceso de } \\
\text { adaptación escolar: } \\
\text { - Integración al centro } \\
\text { - Integración en el grupo } \\
\text { clase. } \\
\text { Desarrollo del conocimien- } \\
\text { to de sí mismo y autono- } \\
\text { mía personal: } \\
\text { - Autonomía física y emo- } \\
\text { - cional } \\
\text { - Autoconcepto y autoes- } \\
\text { - } \text { tima } \\
\text { - eonciencia y regulación } \\
\text { - Valores } \\
\text { Desarrollo de actitudes, } \\
\text { hábitos y valores del } \\
\text { trabajo: } \\
\text { - Orden } \\
\text { - Cuidado/limpieza } \\
\text { - Participación } \\
\text { - Puntualidad } \\
\text { - Desarrollo de tareas } \\
\text { - Trabajo en equipo }\end{array}$ & $\begin{array}{l}\text { Desarrollo del proceso de } \\
\text { acogida y adaptación al } \\
\text { nuevo centro: } \\
\text { - Integración al centro } \\
\text { - Grupo dinámico y parti- } \\
\text { cipativo. } \\
\text { Desarrollo del conocimien- } \\
\text { to de sí mismo: } \\
\text { - Conocimiento de su } \\
\text { situación madurativa } \\
\text { (adolescencia) } \\
\text { - Capacidades y aptitudes } \\
\text { - Autoconcepto y autoes- } \\
\text { tima } \\
\text { - Personalidad } \\
\text { - Madurez personal y vo- } \\
\text { - Intereses y preferencias } \\
\text { - Expectativas } \\
\text { - Valores } \\
\text { - Hábitos de trabajo y es- } \\
\text { - tudio }\end{array}$ & $\begin{array}{l}\text { Desarrollo del proceso de } \\
\text { acogida y adaptación a los } \\
\text { CFGS y a la universidad: } \\
\text { - Proceso de transición } \\
\text { educación secundaria- } \\
\text { educación superior } \\
\text { - Adaptación a los estu- } \\
\text { dios superiores. } \\
\text { Desarrollo de la identidad } \\
\text { personal y profesional: } \\
\text { - Sus experiencias acadé- } \\
\text { micas y profesionales } \\
\text { - Sus concepciones sobre } \\
\text { la institución y los estu- } \\
\text { dios elegidos } \\
\text { - Sus habilidades y com- } \\
\text { petencias (estilos de } \\
\text { aprender y evaluar, in- } \\
\text { teligencia emocional, } \\
\text { creatividad, etc.) } \\
\text { - Su proyecto profesional }\end{array}$ \\
\hline
\end{tabular}




\begin{tabular}{|c|c|c|}
\hline Infantil y primaria & Secundaria & ación superior \\
\hline 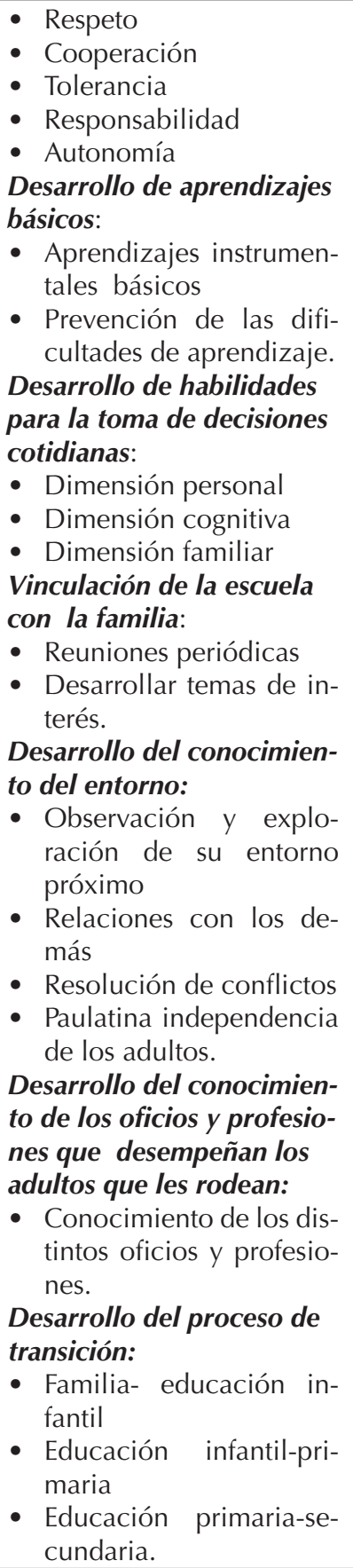 & 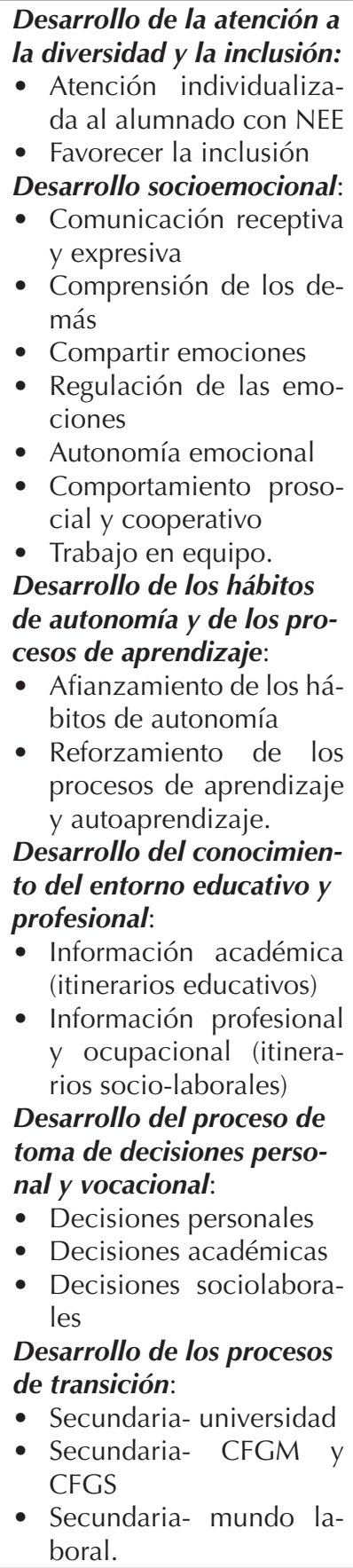 & 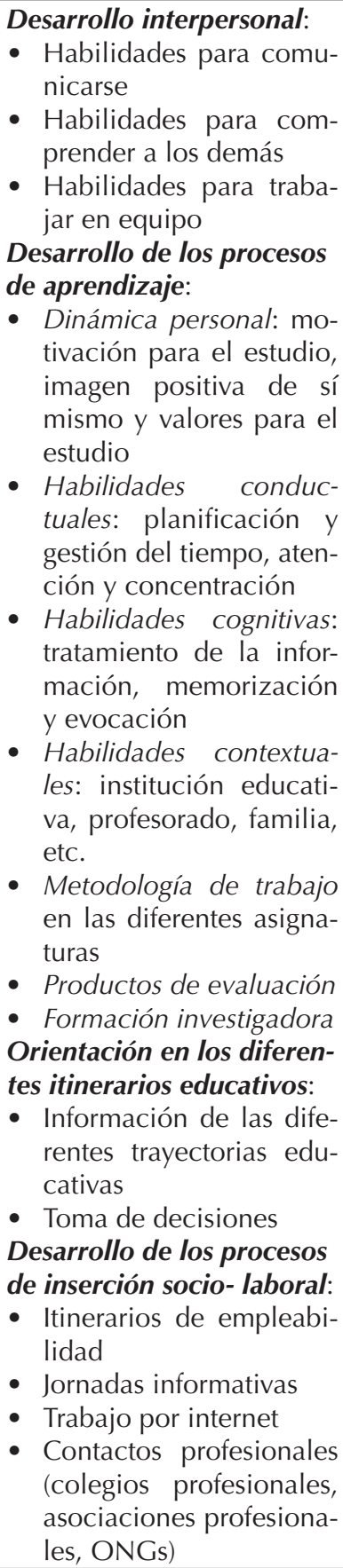 \\
\hline
\end{tabular}




\subsection{Estrategias de intervención}

A la hora de diseñar y poner en marcha la acción tutorial, desde una perspectiva integral, se pueden adoptar diferentes estrategias de intervención en función de: 1) la predisposición e implicación de la institución; 2) la implicación del docente; 3) los recursos humanos y materiales de que se dispone (Álvarez González, 2006, 2009, 2014). Las estrategias más utilizadas las podríamos concretar en:

A) Creación de un currículum específico para la acción tutorial. Se diseña un currículum paralelo donde se desarrolla la acción tutorial en la hora de tutoría. Se contempla en el proyecto educativo del centro (PEC) y en el plan de acción tutorial (PAT), pero no dentro de las diferentes áreas curriculares.

B) Se produce la integración de determinados objetivos de la acción tutorial en algunas de las asignaturas. Esta estrategia es bastante usual, pero ha sido llevada con escaso rigor. Además no todos los objetivos de la acción tutorial pueden ser asumidos en el currículum. No cabe duda que la técnica integradora más práctica es el incorporar los objetivos de la tutoría con los objetivos educativos.

C) Plena integración en la globalidad del currículum. La acción tutorial se va desarrollando a través de las materias escolares y tutorías. La función del especialista es la de consultor, asesor, dinamizador y facilitador de recursos y la del profesor-tutor es la de llevar a cabo la intervención. Cada profesor es un tutor que habrá de abordar la acción tutorial en su materia.

D) Integración de la tutoría de forma interdisciplinar. Se trata de incorporar contenidos de tutoría en las diferentes áreas curriculares de forma transversal. Esto supondría: 1) una perfecta coordinación del profesorado; 2) un compromiso de trabajar de forma interdisciplinar. La dificultad estriba en cómo coordinar e implicar al profesorado de las diferentes materias.

E) Sistema de programas integrados (SPI). Consiste en aglutinar en un solo PAT las diferentes acciones de orientación y tutoría, que se están llevando a cabo en una institución, produciendo con ello en el alumnado y las familias un efecto sinergia. 


\subsection{Modelo organizativo}

Con frecuencia nos olvidamos de aquellos aspectos que hacen posible lo que hemos diseñado y planificado. Detrás de una buena planificación ha de haber una adecuada organización si queremos tener éxito en la intervención (Álvarez González y Álvarez Justel, 2015). Este modelo organizativo ha de tener en cuenta: 1) el ámbito de actuación (aula, centro, sector); 2) los niveles de intervención (la acción tutorial, las Unidades y/o Departamentos de orientación y Servicios de orientación); 3) y el tipo de unidad (directa e indirecta) (Tablas 6 y 7 ).

Tabla 6. Estructura organizativa y funcional de la tutoría en la educación infantil, primaria y secundaria.

\begin{tabular}{lll}
\hline Ámbitos & \multicolumn{1}{c}{ Niveles de intervención } & \multicolumn{1}{c}{ Tipo de unidad } \\
\hline Aula & $\begin{array}{l}\text { Acción tutorial (Tutoría de asignatura, } \\
\text { tutoría de acompañamiento y tutoría de } \\
\text { asesoramiento personal) }\end{array}$ & $\begin{array}{l}\text { Unidad de acción } \\
\text { directa }\end{array}$ \\
\hline Centro & $\begin{array}{l}\text { Unidades de orientación en infantil y } \\
\text { primaria } \\
\text { Departamento de orientación en secun- } \\
\text { daria }\end{array}$ & $\begin{array}{l}\text { Unidad organizativa bá- } \\
\text { sica en el centro (acción } \\
\text { indirecta) }\end{array}$ \\
\hline Sector & Equipos de sector & $\begin{array}{l}\text { Unidad eje en un área } \\
\text { o sector escolar (acción } \\
\text { indirecta). }\end{array}$ \\
\hline
\end{tabular}

Tabla 7. Estructura organizativa y funcional de la tutoría en la educación superior.

\begin{tabular}{|c|c|c|}
\hline Ámbitos & Niveles de intervención & Tipo de unidad \\
\hline $\begin{array}{l}\text { Aula (clase, } \\
\text { pequeño } \\
\text { grupo) }\end{array}$ & $\begin{array}{l}\text { Acción tutorial (Tutoría de asigna- } \\
\text { tura, tutoría de acompañamiento y } \\
\text { tutoría de asesoramiento personal) }\end{array}$ & Unidad de acción directa \\
\hline $\begin{array}{l}\text { Titulación/ } \\
\text { facultad }\end{array}$ & Coordinación de tutoría & $\begin{array}{l}\text { Unidad organizativa bá- } \\
\text { sica en la titulación y la } \\
\text { Facultad (acción indirecta) }\end{array}$ \\
\hline $\begin{array}{l}\text { Universi- } \\
\text { dad }\end{array}$ & $\begin{array}{l}\text { Servicios de información, formación } \\
\text { y orientación de soporte a la tutoría }\end{array}$ & $\begin{array}{l}\text { Unidad organizativa en } \\
\text { la Universidad (acción } \\
\text { indirecta). }\end{array}$ \\
\hline
\end{tabular}




\section{A modo de conclusión}

Se ha de tender hacia un modelo integrador de la tutoría en los diferentes niveles educativos, afrontando el desarrollo integral del alumnado en todas sus dimensiones (personal, socioemocional, académica y profesional). La tutoría se ha de convertir en un factor de calidad de la educación y así producir un verdadero efecto sinergia en el alumnado. Para ello, se ha de reforzar la función tutorial, como algo inherente y fundamental de la función docente: todo profesor es tutor. Todo esto va a suponer una acción coordinada por parte de todas las personas e instituciones implicadas en el proceso formativo; una programación de la tutoría, adaptada a las necesidades de los usuarios; la implicación, motivación y formación del docente; y la ayuda, el soporte técnico del especialista de orientación del centro y de los diferentes servicios que ofrece la comunidad.

\section{Referencias bibliográficas}

Albaigés, B., y Martínez, M. (2013). L'estat de l'educació a Catalunya. Anuari 2013. Barcelona: Fundació Jaume Bofill.

Álvarez González, M. (2004). La acción tutorial como factor de calidad de la educación. En A. del Valle (coord.) .Contextos educativos y acción tutorial (pp. 71-109). Madrid: MEC.

Álvarez González, M. (2006). Planificación y organización de la acción tutorial. En M. Álvarez González (coord.). La acción tutorial: su concepción y su práctica (pp. 2780). Madrid: MEC.

Álvarez González, M. (2008). La tutoría académica en el EEES. Revista Interuniversitaria de Formación del Profesorado, 22(1), 71-88.

Álvarez González, M. (2009). Planificación y organización de la tutoría en la Universidad. En M. Álvarez González y R. Bisquerra (coords.). Manual de orientación y tutoría (Formato CD). Madrid: Wolters Kluwer Educación.

Álvarez González, M. (2014). Hacia un modelo integral de tutoría universitaria. En M. Álvarez González y R. Bisquerra (Coords.). Manual de orientación y tutoría (Versión electrónica). Madrid: Wolters Kluwer Educación.

Álvarez González, M., y Rodríguez Espinar, S. (2000). Cambios socioeducativos y orientación en el Siglo XXI: Nuevas estructuras, roles y funciones. En M. AA.VV. XII Congreso Nacional y I Iberoamericano de Pedagogía (Tomo I. Pp- 637- 686). Madrid: Sociedad Española de Pedagogía.

Álvarez González, M. (coord.) (2006). La acción tutorial: su concepción y su práctica. Madrid: MEC.

Álvarez González, M., y Forner, A. (coords.) (2008). La tutorial universitària: Guia pràctica. Barcelona: ICE Universidad de Barcelona (Formato CD). 
Álvarez González, M., y Álvarez Justel, J. (2013). Dimensiones y competencias clave en la formación inicial y permanente del orientador. En M. Álvarez y R. Bisquerra (Coords.). Manual de orientación y tutoría (Versión electrónica). Manual de orientación y tutoría (Versión electrónica). Madrid: Wolters Kluwer Educación.

Álvarez González, M., y Álvarez Justel, M. (2014). La orientación y la tutoría en los postgrados. En M. Álvarez González y R. Bisquerra (Coords.). Manual de orientación y tutoría (Versión electrónica). Madrid: Wolters Kluwer Educación.

Álvarez González, M., y Ávarez Justel, J. (2015). La tutoría universitaria. Del modelo actual a un modelo integral. Revista Electrónica Interuniversitaria de Formación del Profesorado (REIFOP), 18(2), 125-142.

Álvarez González, M., y Álvarez Justel, J. (2016). Educación para la carrera en educación infantil y primaria. Revista de Orientación Educacional, 30 (58), 8-34.

Álvarez Pérez, P. (2002). La función tutorial en la universidad: Una apuesta por la mejora de la calidad de la enseñanza. Madrid: EOS.

Álvarez Pérez, P., González, M. C. (2009). Modelo comprensivo para la institucionalización de la orientación y la tutoría en la enseñanza universitaria. Revista Qurriculum, 22, 73-95.

Arbizu Bakaikoa, F., Lobato Fraile, C., y del Castillo, I. (2005). Algunos modelos de abordaje de la tutoría universitaria. Revista de Psicodidáctica, 10 (1), 7-21.

Arias Aparicio, F., y Gentile, A. (2011). Calidad y reforma de la ESO en España. Madrid: Fundación Alternativas.

ASCA (2005).Tennessee Model for Comprehensive School Counseling. The School Counseling Framework. Recuperado de: https://www.tn.gov/assets/entities/education/attachments/comp_sch_counsel_model.pdf

Barna, J., and Brott, P. (2011). How Important is Personal Social Development to Academic Achievement?. The Elementary School Counselor's Perspective Professional. School Counseling, 14 (3), 242-249.

Braslavsky, C. (2004). Diez factores para una educación de calidad para todos en el S. XXI. Madrid: Fundación Santillana.

Burgess, D. G. (1994). The Educational Quality Improvement Process Model.En D. G. Burgess y R. M. Dedmond (Eds.). Quality Leardership and The Professional School Counselor. Alexandria: American School Counselor Association.

Comisión Europea (2000). Informe europeo sobre la calidad de la educación. Diecisiete indicadores de calidad. Revista Española de Educación Comparada, 6, 389-394.

Comunidad de Madrid. Consejería de Educación (2008). Currículum de educación infantil: conocimiento de sí mismo y la autonomía personal. BOCM, nº 61 del 12 de marzo.

Connecticut State Departament of Education (2014).Connecticut Early Learning and Development Standards (CTELDS). Recuperado de: http://www.ct.gov/oec/cwp/view. asp? $\mathrm{a}=4541 \& \mathrm{q}=536726$.

De la Cruz Flores, G., García Campos, T., y Abreu Hernández, L. F. (2006). Modelo integrador de la tutoría. Revista Mexicana de Investigación Educativa, 11 (31), 1363-1388.

De Miguel, M. (1997). La evaluación de los centros. Actas de las VII Jornadas sobre la LOGSE. Facultad de Ciencias de la Educación. Universidad de Granada.

Delors, J. (1996). La educación encierra un tesoro. Madrid: UNESCO-Santillana. 
Hacia un modelo integrador de la tutoría en los diferentes niveles educativos Manuel Álvarez González

García Pérez, S. L. (2010). El papel de la tutoría en la formación integral del universitario. Tiempo de Educar, 21, 31-56.

Gergiannis, V. C., y Fitsilis, P. (2010). Evaluation of the Factors that Determine Quality in Higher Education.AnEmpiricalStudy. Recuperado de: http://www.emeraldinsigh. com/0968-4883.htm.

Ginter, Z. J., Salise, J. J., y Press, N. (1990). The Elementary School Counselor's Role: Perceptions of Teachers. The School Counselor, 38, 19-23.

Gobierno de Navarra (2001). La orientación educativa en educación infantil y primaria. Pamplona: Ona. Recuperado de: http://dpto.educacion.navarra.es/publicaciones/pdf/ orien_educ.pdf

González Bueno, G., Bello, G., y Arias, M. (2012). El impacto de la crisis en los niños. La infancia en España. Madrid: UNICEF. Recuperado de: http://www.unicef.es/actualidad-documentacion/publicaciones/la-infancia-en-espana-2012-2013.

González Palacios, A., y Avelino Rubio, I. (2016). Tutoría: Una revisión conceptual. Revista de Educación y Desarrollo, 38, 57-68.

Hamlin, D., y Kidder, A. (2015). Guiding Students to Success. Ontario's School Guidance. Toronto: People for Education. Recuperado de: http://www.peopleforeducation.ca/ wp-content/uploads/2015/01/guidance-2015-WEB.pdf.

Hill, P. L. (2012). Comprehensive Developmental School Counseling Model Refence Guide. West Virginia Higher Education Policy Commission. Recuperado de: http://wvde. state.wv.us/counselors/documents/wvsc_model_booklet_colorJuly30_2012.pdf

Hillsdale, N. J. (2012). Career Awareness Curriculum Kindergarten-Grade Eight. Hillsdale Public Schools.

LOGSE (1990). Ley Orgánica de Ordenación General del Sistema Educativa. Madrid: MEC.

LOPEG (1995). Ley Orgánica de Participación, Evaluación y Gobierno de los Centros. Madrid: MEC.

LOCE (2002). Ley Orgánica de Calidad de la Educación. Madrid: MEC.

LOE (2006). Ley Orgánica de Educación. Madrid: MEC.

LOMCE (2013). Ley Orgánica para la mejora de la Calidad de la educación. Madrid: MEC.

LOU (2001, 2007). Ley Orgánica de Universidades. Madrid: MEC.

Marín, M. A., y Rodríguez Espinar, S. (2001). Prospectiva del diagnóstico y la orientación. Revista de Investigación Educativa, 19 (2), 315-362.

McLellan, R., and Galton, M. (2015).The Impact of Primary-Secondary Transition Students. Recuperado de: http://www.nuffieldfoundation.org/sites/default/files/files/ McLellan\%20-\%20Final-Report-June-2015.pdf

McQuillan, M. K., y Coleman, G. A. (2008). Comprehensive School Counseling: A Guide to Comprehensive School Counseling Program Development. Departmen of Education State of Connecticut. Recuperado de: http://www.sde.ct.gov/sde/lib/sde/PDF/ DEPS/Special/counseling.pdf

Montserrat Pera, M. S. (2011). La tutoria en els procesos d'ensenyament.aprenentatgedesenvolupats a través d'entorns virtuals d'aprenentatge. Tesis Doctoral. UniversitatRoviriiVirgili. Tarragona.

NACADA (2008). Making Career Advising Integral to Academic Advising. Recuperado de: http://www.nacada.ksu.edu/Resources/Product-Details/ID/REC035CD.aspx 
Pantoja, A. (2006). Acción tutorial y nuevas tecnologías. En M. Álvarez González (Coord.). La acción tutorial: su concepción y su práctica (pp. 219-261). Madrid: MEC. Pantoja. A. (2010). El orientador ante las nuevas tecnologías. Recuperado de: http://www. hdl.handle. Net/10272/3449.

Public Schools of North Carolina (2001).Guidance Curriculum for the Comprehensive School Counseling Program K-12. State Board of Education. Departmen of Public Instruction. Recuperado de: http://www.dpi.state.nc.us/docs/curriculum/guidance/ resources/programs-study.pdf

Rodríguez Espinar, S. (1995). Un reto profesional: la calidad en la intervención orientadora. En R. Sanz, F. Castellano y J. A. Delgado (Eds.). Tutoría y orientación (pp. 119135). Barcelona: Cedecs.

Rodríguez Espinar, S. (2002). La orientación y la función tutorial, factores de la calidad de la educación. En CES (Ed.). Los educadores en la sociedad del S. XXI (pp. 89-160). Madrid: MEC.

Rodríguez Espinar, S. (coord.) (2004). Manual de tutorial universitaria. Barcelona: Octaedro: ICE- UB.

Rodríguez Espinar, S. (2006). Función tutorial y calidad de la educación. En M. Álvarez González (Coord.). La acción tutorial: su concepción y su práctica (pp. 9-25). Madrid: MEC.

Rodríguez Espinar, S. (2015). La evaluación de la calidad de la educación obligatoria. Madrid: Síntesis.

Sanz, R. (2001). Orientación Psicopedagógica y calidad educativa. Madrid: Pirámide.

Sanz, R. (2010). El profesor como tutor: un reto a consolidar en el ejercicio profesional de la orientación. Revista Española de Orientación y Psicopedagogía, 22 (2), 346-357.

Sanz, R., y Sobrado, L. (1998). Roles y funciones de los orientadores. Revista de Investigación Educativa, 16 (2), 25-57.

Sarramona, J. (2002). Desafios a la escuela del siglo XXI. Barcelona: Octaedro.

Stevenson, N. (2006). Integrating Personal Tutoring with Personal Development Planning. University of Westminster. Recuperado de: https://www.heacademy.ac.uk/system/ files/web0150_ecasebook_intergrating_personal_tutoring_with_personal_development_planning_0.pdf

Texas Public School (2004).A Model Comprehensive Developmental Guidance and Counseling Program PRE-K-12 Grade. Recuperado de:http://int.search.myway.com/ search/GGmain.jhtml? searchfor=integrated+system+guidance+in+primary+and+se cundary+school \&n=782acb0c\&p2=\%5EBVA\%5Exdm067\%5ETTAB02\%5Ees\&ptb= 6B197785-58B3-44EC-AE7A-57C4AD333D35\&qs=\&si=CJ6lvZzH2c0CFTUo0wod5 IQHgQ\&ss=sub\&st=tab\&trs=wtt\&tpr=sbt\&ts=1482405069402

Thayer, Y. V. (1994). Total Quality Management: How it Works in School. En D. G. Burgess y R. M. Dedmond (Eds.). Quality Leadership and The Professional School Counselor. Alexandria: American School Counselor Association.

The Education Bureau HKSAR (2006). Guide to the Pre-Primary Curriculum PRE- Primary C. The Curriculum Development Council. Recuperado de: http://www.edb.gov. hk/attachment/en/edu-system/preprimary-kindergarten/overview/pre-primaryguidenet_en_928.pdf 
Hacia un modelo integrador de la tutoría en los diferentes niveles educativos

Manuel Álvarez González

Tracey, M., y Shea, B. (Eds.) (2000). Career Education in the Elementary School. St. John's: University of Newfoundland.

UNESCO (2004). 47ª Reunión de la Conferencia Internacional de Educación (CIE). Recuperado de: http://www.unesdoc.org/images/0015/001530/153069s.pdf.

UNESCO (2014). Enseñanza y aprendizaje: Lograr la calidad para todos. Recuperado de: http://www.unesdoc.org/images/0022/002261/226159s.pdf.

UNESCO (2015). La educación para todos, 2005-2015: Logros y desafíos. Recuperado de: http://www.unesdoc.org/images/0023/002324/232435s.pdf.

UNICEF (2013). El impacto de la crisis en los niños. Madrid: UNICEF España.

Watts, A. G., y Esbroeck, R. V. (1998). News Skills for News Futures. Bruselas: VUBPRESS.

Welch, I. D., y McCarrol, L. (1993). The future Role of School Counselors. The School Counselor, 41, 49-53. 